\title{
The Identification of Stenotrophomonas maltophilia Contamination in Ultrasound Endoscopes and Reproduction of Decontamination Failure by Deliberate Soiling Tests
}

\author{
Jos A. Stigt ${ }^{a}$ Maurice J. Wolfhagen ${ }^{b}$ Patricia Smulders ${ }^{c}$ Vera Lammers $^{a}$ \\ Departments of a Pulmonology, ${ }^{\mathrm{b}}$ Clinical Microbiology and Infectious Diseases and ${ }^{\mathrm{c}}$ Hygiene and Infection Prevention, \\ Isala Hospital, Zwolle, The Netherlands
}

\section{Key Words \\ Stenotrophomonas maltophilia. Pseudo-infection . \\ Endoscopic ultrasound - Endobronchial ultrasound . \\ Disinfection · Contamination - Endoscope drying cabinet}

\begin{abstract}
Background: An outbreak of Stenotrophomonas maltophilia was observed in the cultures of bronchial aspirations. After systematic culturing of the scopes and reprocessing the equipment, it turned out to be a pseudo-outbreak caused by a failure of the disinfector to sterilize ultrasound scopes, subsequently resulting in cross infection of the bronchoscopes via the connecting tubes in the dryer. Objectives: To support the above-mentioned findings and to show how different variables influence the decontamination process. Methods: The ultrasound scopes were deliberately contaminated with test soil and subsequently disinfected with peracetic acid (PAA), glutaraldehyde (GA) and hot water. Cultures were taken immediately after disinfection and after 4 days in the drying cabinet. Results: After disinfection with PAA, persistent contamination was observed in the endobronchial ultrasound scope, and after disinfection with PAA and prolonged storage in the drying cabinet, contamination of the endoscopic ultrasound scope persisted. All ultrasound scopes were effectively disinfected with GA. Conclusion: It is con-
\end{abstract}

cluded that disinfection with PAA fails to decontaminate the small channels of the ultrasound scopes. Prolonged storage in humid conditions seems to enhance the outgrowth of microorganisms. By reassignment of all endoscopes to fixed locations in the drying cabinet, by using disposable connector tubes for the balloon channels, and by using GA instead of PAA, the reprocessing procedure in our hospital was corrected effectively.

(c) 2015 S. Karger AG, Basel

\section{Introduction}

Current pulmonology practice shows an increased use of endobronchial ultrasound (EBUS) and endoscopic ultrasound (EUS). Unlike bronchoscopy, which is used to diagnose both microbiological and malignant disease, endoscopic ultrasound techniques are mainly used to diagnose and stage cancer.

Disinfection of endoscopes is essential to prevent cross infection from patient to patient. To enable safe reuse, contaminated endoscopes are reprocessed according to the guidelines on cleaning and disinfection issued by the British Society of Gastroenterology Endoscopy Committee $[1,2]$. In order to prevent transmission of Gram-negative bacteria from patient to patient by contaminated

\section{KARGER 125}

C 2015 S. Karger AG, Base

0025-7931/15/0896-0565\$39.50/0 


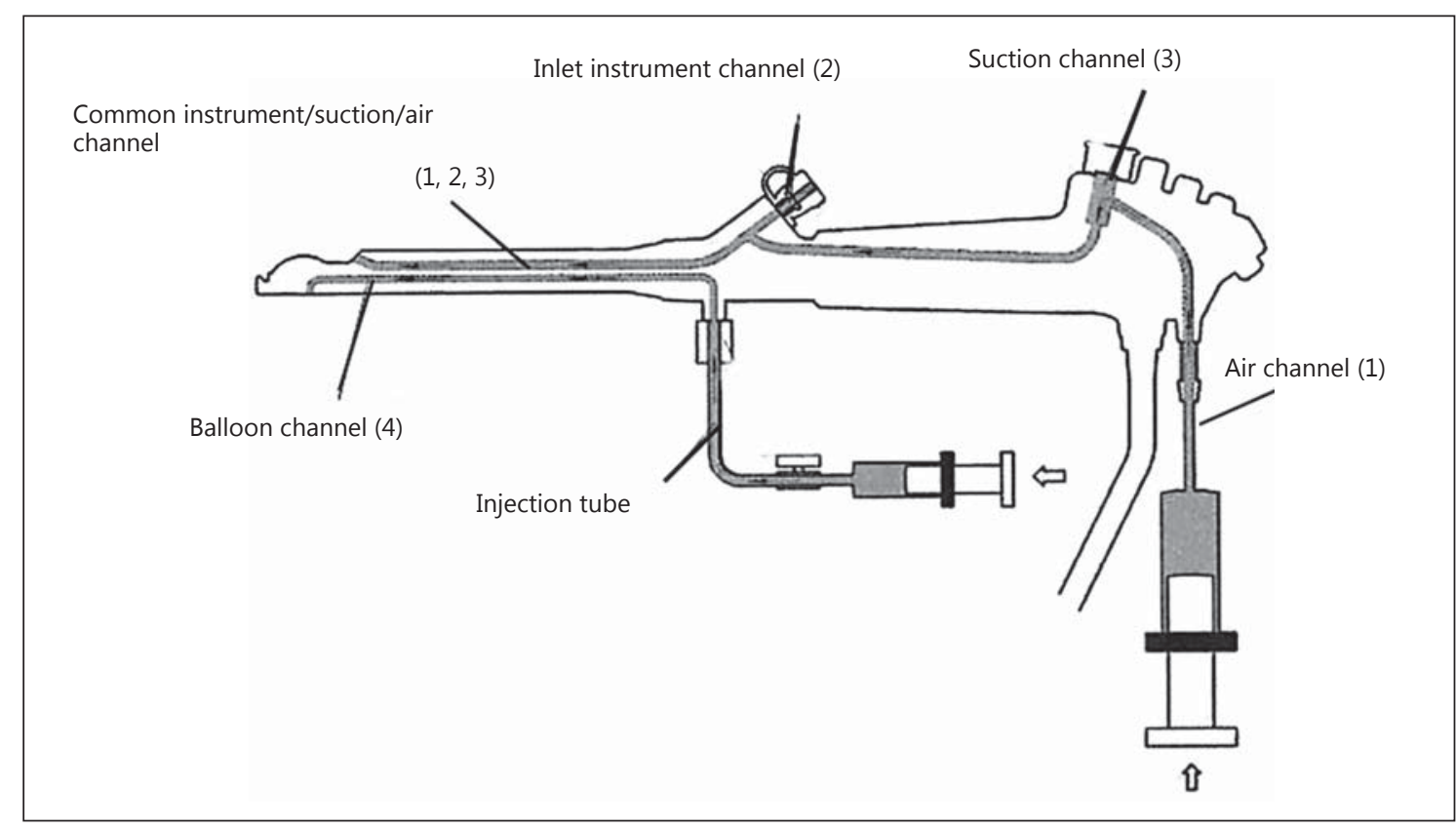

Fig. 1. Cross section of an EBUS scope. The numbers designating the channels of the scope correspond to the numbers of the channels in table 1.

scopes, routine microbiological sampling of rinse water during reprocessing was proposed [3].

In the Netherlands, a steering committee provided a meticulous description of the disinfection and safety procedures as well as stepwise measures to be taken after incidents [4]. According to these guidelines, the final rinse water of disinfectors should be cultured for aerobic mesophilic bacteria 4 times a year so as to detect inadequate disinfection. Moreover, an annual inspection of scopes, connectors and channel separators is mandatory; however, routine cultures of scopes are not required.

In our hospital we found that the standard screening procedure was not sufficient to detect contamination after reprocessing of the bronchoscopes and ultrasound endoscopes. Three positive cultures (from two different bronchoscopes) with Stenotrophomonas maltophilia on 3 days (7-9 September 2011) in the bronchial aspirations of 3 different patients alarmed the clinical microbiologist, since this finding suggested a possible contamination of our endoscopes. When the cultures of bronchoscopic aspirates were compared with sputum cultures positive for S. maltophilia, a temporary fluctuation in the incidence of $S$. maltophilia was considered unlikely.

Consequently, cultures taken from all endoscopes tested positive for S. maltophilia, as did the tubes that connected the endoscopes to the drying cabinet.
After disinfection with peracetic acid (PAA), the bronchoscopes became sterile. However, the ultrasound endoscopes remained contaminated, even after three disinfection cycles with PAA; subsequent disinfection of the ultrasound scopes with glutaraldehyde (GA, at a higher temperature than PAA) proved to be effective.

Furthermore, we found that the humidity of the air blown through the channels in the dryer was too high when compared to the recommendations of the manufacturer of the drying cabinet. This conclusion was based on the rapid rate of saturation of the hydro beads that filter the compressed air. Inadequate drying promotes the outgrowth of residential microorganisms in insufficiently disinfected endoscopes.

It was reconstructed that the ultrasound endoscopes contaminated the connecting tubes in the dryer. The bronchoscopes were subsequently cross-infected by connecting them to the same contaminated tubes.

The objectives of this study were (1) to demonstrate the failure of decontamination of ultrasound scopes in our routine cleaning process by reproducing inadequate disinfection after deliberate soiling and (2) to study the effects of an alternative disinfection agent, a higher disinfection temperature and a prolonged storage after disinfection. 


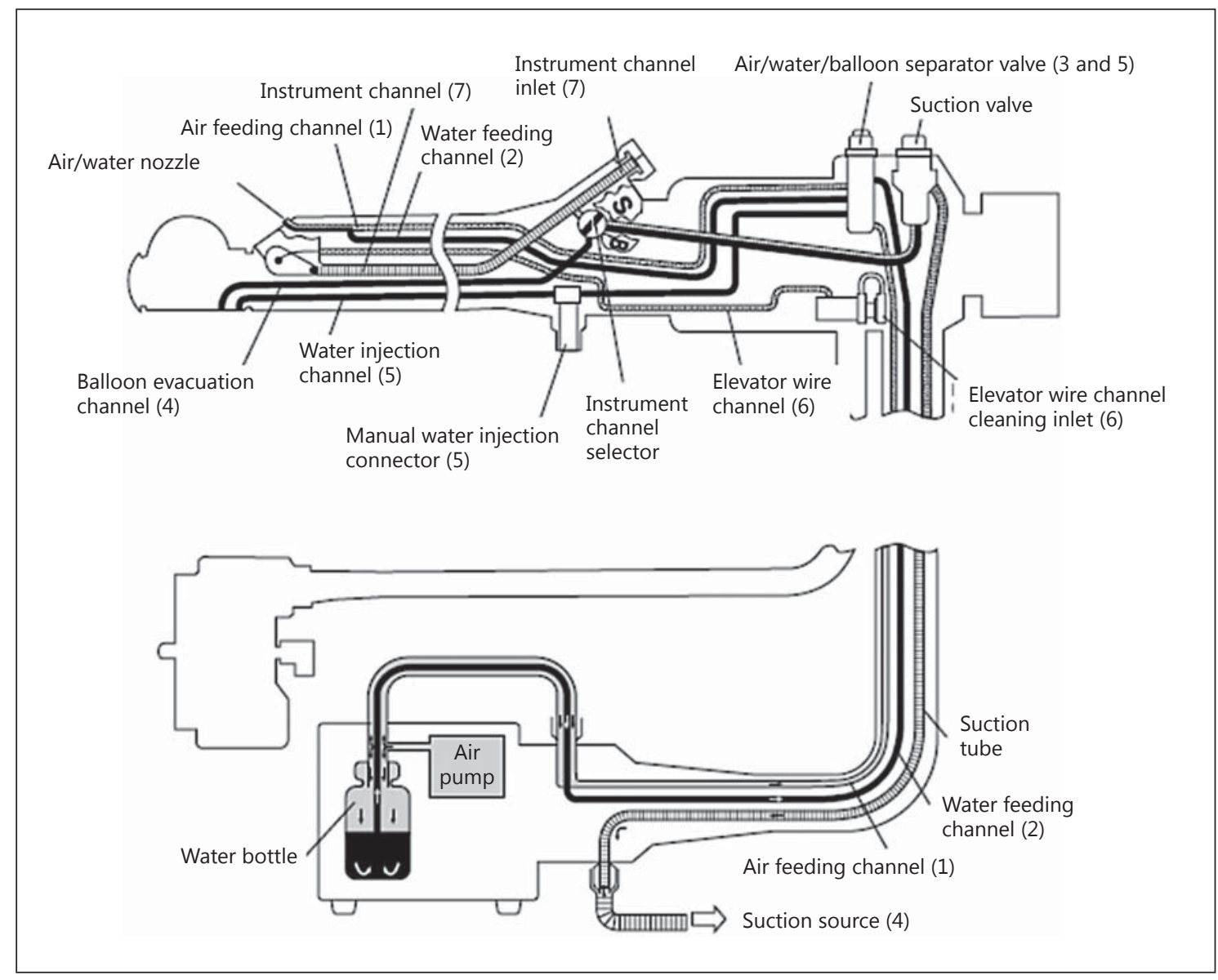

Fig. 2. Cross section of an EUS scope. The numbers designating the channels correspond to the numbers of the channels in table 1.

\section{Methods}

\section{Routine Cleaning of Ultrasound Scopes}

After use, the suction channels of ultrasound scopes are flushed with water. Also, the bigger channels (working and suction channels) are cleaned by a pull-through brush. Furthermore, all channels (including balloon and elevator channel) are flushed with a cleaning solution (MediClean forte 0.5-1.0\%; Dr. Weigert, Assen, The Netherlands). An additional connector (consisting of a tube and metal coupling piece) is used to rinse the balloon channel. The exterior surface of the scopes is brushed and wiped clean with gauze pads.

The basic layouts of the EBUS and EUS scopes are shown in figures 1 and 2, respectively. The tag numbers designating the channels of the scopes correspond to the numbers of the channels in table 1.

\section{Disinfection of Ultrasound Scopes}

In our regular practice, the scopes were transferred to disinfector A (WD440PT; Wassenburg Medical Devices, Dodewaard, The Netherlands) for cleaning with MediClean forte $0.5-1.0 \%$ for 5 min at a temperature of $36-41^{\circ} \mathrm{C}$, followed by disinfection for 10 min with PAA at a temperature of $25-35^{\circ} \mathrm{C}$ (Neodisher Septo PAC $1 \%$; Dr. Weigert).

S. maltophilia Contamination in Ultrasound Endoscopes
When decontamination failure was discovered, the scopes were disinfected temporarily with GA (Disinfectant ETD, Olympus, Hamburg, Germany) in disinfector B (ETD2; Olympus, Tokyo, Japan) for $5 \mathrm{~min}$ at a temperature of $57-62^{\circ} \mathrm{C}$.

Finally the scopes were disinfected in disinfector C. This was exactly the same disinfector as disinfector $\mathrm{A}$, but in this machine the scopes were treated with GA at a temperature of $57-62^{\circ} \mathrm{C}$ for $5 \mathrm{~min}$.

Whenever the scopes are not used within the next $4 \mathrm{~h}$, they are stored in the drying cabinet (GV700, van Vliet Medical Supply, Almere, The Netherlands) for a maximum of 30 days. During storage, all channels of the scopes are connected by tubes to a compressed-air system. The compressed air is led through three filters, including a bacterial filter, and then dried by hydro beads at room temperature. The drying cabinet is maintained at overpressure.

\section{Cultures of Scopes, Devices and Materials}

All endoscopes used in our endoscopy department - two bronchoscopes (EB1970AK and EB1970TK; Pentax, Tokyo, Japan), one endoscopic ultrasound scope (EUS FG-36UX; Pentax) and two endobronchial ultrasound scopes (EBUS EB-1970UK; Pentax) - were cultured by flushing $20 \mathrm{ml}$ saline through the dif- 
Table 1. Culture results of deliberate soiling tests

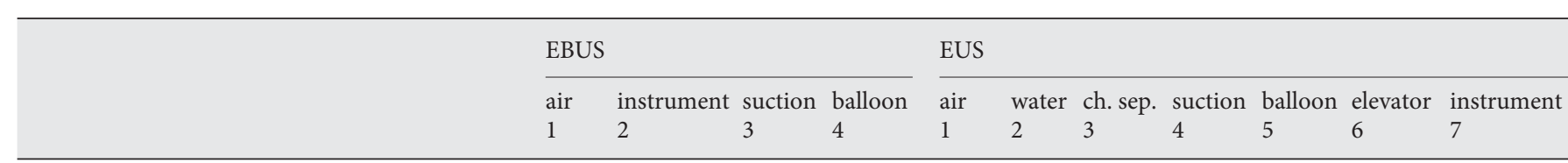

Culture results of deliberately soiled ultrasound endoscopes after disinfecting with $\mathrm{GA}$ at $56-62^{\circ} \mathrm{C}$

After incubation, $\mathrm{CFU} / \mathrm{ml} \times 10^{5}$

After $1 \times$ disinfection with GA, CFU/20 ml

After $2 \times$ disinfection with GA, CFU $/ 20 \mathrm{ml}$ Test soil $150 \times 10^{5}$

Test soil $130 \times 10^{5}$

$\begin{array}{lllllllllll}4.7 & 6.4 & 4.0 & 8.3 & 12.0 & 13.0 & 5.9 & 23.0 & 2.3 & 6.8 & 6.4 \\ 0 & 17^{\mathrm{a}} & 0 & 0 & 0 & 0 & 0 & 0 & 1 & 1 & 0 \\ 0 & 0 & 0 & 0 & 0 & 0 & 1 & 0 & 0 & 0 & 1 \\ 0 & 0 & 0 & 1^{\mathrm{a}} & 0 & 0 & 0 & 0 & 0 & 1 & 3\end{array}$

After $3 \times$ disinfection with GA, CFU/20 ml

Culture results of deliberately soiled ultrasound endoscopes after disinfecting with $G A$ at $56-62^{\circ} \mathrm{C}$ and 4 days in the dryer Test soil $150 \times 10^{5}$

After incubation, $\mathrm{CFU} / \mathrm{ml} \times 10^{5}$

After $1 \times$ disinfection with GA, CFU $/ 20 \mathrm{ml}$

$\begin{array}{lllllll}3.0 & 2.8 & 1.7 & 3.0 & 0.1 & 3.0 & 3.0\end{array}$

After $2 \times$ disinfection with GA, CFU/20 ml

$\begin{array}{lll}3.0 & 2.8 & 1.7 \\ 4^{\mathrm{a}, \mathrm{b}} & 0 & 1^{\mathrm{a}}\end{array}$

0
$<20$

$\begin{array}{lll}0.1 & 3.0 & 3.0 \\ 1^{\mathrm{a}} & 1^{\mathrm{a}, \mathrm{b}} & 2^{\mathrm{a}}\end{array}$

$\begin{array}{llll}0 & 0 & 0 & <20\end{array}$

$<20 \quad 0 \quad 0$

Culture results of deliberately soiled ultrasound endoscopes after washing with water at $56-62^{\circ} \mathrm{C}$ without disinfectant Test soil $180 \times 10^{5}$

After incubation, $\mathrm{CFU} / \mathrm{ml} \times 10^{5}$

After $1 \times$ washing, CFU/20 ml

After $2 \times$ washing, CFU/20 ml

After $1 \times$ disinfection with GA, CFU/20 ml

$\begin{array}{lllllllllll}4.4 & 3.8 & 6.4 & 4.7 & 24.0 & 14.0 & 75.0 & 21.0 & 10.7 & 5.6 & 48.0 \\ 0 & 0 & 1^{\mathrm{d}} & 0 & 0 & 1 & 0 & 2 & 0 & 73 & 1 \\ 4^{\mathrm{a}} & 0 & 0 & 0 & 0 & 0 & 1 & 1 & 0 & 4 & 1^{\mathrm{a}} \\ 0 & 0 & 0 & 0 & 1^{\mathrm{a}} & 0 & 1^{\mathrm{e}} & 0 & 3^{\mathrm{a}} & 1^{\mathrm{a}} & 0\end{array}$

Culture results of deliberately soiled ultrasound endoscopes after disinfecting with $P A A$ at $25-35^{\circ} \mathrm{C}$ and finally $\mathrm{GA}$

After incubation, $\mathrm{CFU} / \mathrm{ml} \times 10^{5}$

After $1 \times$ disinfection with PAA, CFU/20 ml

After $1 \times$ disinfection with PAA, CFU/20 ml

After $1 \times$ disinfection with GA, CFU/20 ml

Test soil $130 \times 10^{5}$

Test soil $140 \times 10^{5}$

$\begin{array}{lllllllllll}4.2 & 8.6 & 4.0 & 4.1 & 8.2 & 7.1 & 6.8 & 32.0 & 18.0 & 4.1 & 14.0 \\ 0 & 1 & 0 & 27 & 1 & 0 & 0 & 0 & 0 & 0 & 0 \\ 0 & 0 & 0 & 0 & 0 & 0 & 0 & 0 & 0 & 0 & 0 \\ 0 & 0 & 0 & 0 & 0 & 0 & 8^{\mathrm{a}} & 0 & 0 & 0 & 0\end{array}$

Culture results of deliberately soiled ultrasound endoscopes after disinfecting with $\mathrm{PAA}$ at $25-35^{\circ} \mathrm{C}$ and 4 days in the dryer and finally $\mathrm{GA}$ at $56-62^{\circ} \mathrm{C}$

After incubation, $\mathrm{CFU} / \mathrm{ml} \times 10^{5}$

After $1 \times$ disinfection with PAA, CFU/20 ml

After $1 \times$ disinfection with GA, CFU/20 ml Test soil $90 \times 10^{5}$

$\begin{array}{lllllllllll}6.8 & 9.0 & 8.9 & 3.2 & 21.0 & 6.8 & 8.7 & 31.0 & 5.8 & 5.5 & 8.8 \\ 7^{\mathrm{c}} & 0 & 0 & 0 & 300 & 200 & 330 & 20 & 100 & 80 & 1^{\mathrm{f}} \\ 0 & 4^{\mathrm{b}} & 0 & 1^{\mathrm{g}} & 0 & 2^{\mathrm{b}} & 0 & & 0 & 0 & 0\end{array}$

Numbers without a superscript refer to $S$. maltophilia. ch. sep. $=$ Channel separators. ${ }^{\text {a }}$ Gram-positive cocci. ${ }^{b}$ Gram-positive cocci and also $3 \mathrm{CFU} / 20 \mathrm{ml} \mathrm{Gram}$-negative cocci. ${ }^{\mathrm{c}} \mathrm{Gram}$-positive cocci and also $1 \mathrm{CFU} / 20 \mathrm{ml}$ Aspergillus fumigatus. ${ }^{\mathrm{d}}$ Microorganisms but no S. maltophilia. ${ }^{\mathrm{e}}$ Moraxella species. ${ }^{\mathrm{f}}$ Staphylococcus pasteuri. ${ }^{\mathrm{g}}$ Gram-negative rods, no S. maltophilia.

ferent channels; this is in concurrence with the guidelines described in the Dutch Quality Handbook for flexible endoscopy, cleaning and disinfection version 2.0, 2010, written by the Steering Committee Flexible Endoscopy Cleaning and Disinfection (Stuurgroep Flexibele Endoscopen Reiniging en Desinfectie). The specimens were then filtered through a $0.22-\mu \mathrm{m}$ membrane before being placed on a R2A-agar plate. After incubating the cultures for 5 days at $28-32^{\circ} \mathrm{C}$, an estimation of biological growth was made, expressed in colony-forming units (CFUs). Cultures with at least 20 CFUs per $20 \mathrm{ml}$ were considered significant.

The manufacturer checked the ultrasound scopes on location, but considered an inspection of the interior of the scopes superfluous. Leak tests were always negative. The drying cabinet was also checked by its manufacturer.

Disinfector A with all its components and connecting tubes and the drying cabinet with its connecting tubes as well as the rinse water and detergent were cultured.

\section{Cultures after Deliberate Contamination}

Cultures as described earlier were taken after deliberate contamination of all channels of the ultrasound scopes with test soil containing S. maltophilia ST16 (the strain repeatedly isolated from the contaminated endoscopes).

The soil was prepared by inoculating S. maltophilia ST16 in a pasteurized human plasma protein solution of $4 \%$ albumin before culturing overnight at $35^{\circ} \mathrm{C}$. The next morning, the broth was cultured quantitatively to determine colony-forming units per millilitre and flushed through all channels of the endoscope until all channels were filled. Then the scope was left at room temperature overnight with the test soil in all channels. The next day air was blown through all channels to remove the test soil and cultures were taken according to the previously mentioned method. The contaminated scopes were disinfected by two successive cycles with PAA in disinfector A, followed by a definitive cycle with GA in disinfector $\mathrm{C}$, or by three successive cycles with GA. Temperatures and cleaning times were used as described earlier. To study 
the effectiveness of flushing with just hot water (without disinfectant), all channels were flushed twice with water (in disinfector C) at $57-62^{\circ} \mathrm{C}$ followed by a final disinfection cycle with GA in disinfector $\mathrm{C}$, after which they were cultured. All disinfecting and cleaning procedures were repeated twice.

A second series of soiling tests was performed for all channels, however, this time after the scopes had been placed in the dryer for 4 days, so as to imitate the clinical situation in which a scope was used after a protracted interval (for example after a weekend), and as such provoking a potential outgrowth of small colonies of microorganisms. All cultures with dilution series were performed twice.

\section{Results}

The variables tested in EUS and EBUS endoscopes were: disinfectant (PAA and GA) and temperature. Table 1 shows the culture results after deliberately soiling the ultrasound endoscope channels with S. maltophilia and after disinfecting the channels with GA. No significant growth of cultures was seen after GA disinfection, and after 4 days of storage following GA disinfection, no positive cultures were found.

Table 1 shows the culture results after flushing with hot water without disinfectant. The elevator channel of the EUS endoscope was not disinfected by using just hot water, but all EBUS endoscope cultures turned out negative after flushing with hot water. A few microorganisms grew at non-significant levels in cultures taken from rinse water.

The culture results after deliberate soiling followed by disinfecting with PAA are shown in table 1. The EBUS endoscope balloon channel remained contaminated after one disinfection cycle. After 4 days of storage following PAA disinfection, the EUS endoscopes showed positive cultures; however, the EBUS endoscopes did not. No $S$. maltophilia was cultured from the rinse water used.

\section{Discussion}

\section{Disinfection Process}

Inadequate Disinfection of Ultrasound Endoscopes

In the reprocessing setting described, disinfection of $\mathrm{E}(\mathrm{B}) \mathrm{US}$ scopes is inadequate when PAA is used as a disinfectant. This was shown by the high number of persisting colony-forming units in cultures from small channels. The balloon channel of the EBUS was not adequately disinfected with PAA, and the elevator channel of the EUS was not adequately disinfected by hot water; also, disinfection with PAA was not adequate after 4 days of storage. Presumably the disinfecting agent cannot reach all areas of the ultra- sound scopes sufficiently, due to the formation of niches caused by differences in temperature and pressure.

In the end, inadequate decontamination of ultrasound endoscopes resulted in pseudo-infections detected in samples acquired with cross-infected bronchoscopes. Pseudo-infections from ultrasound scopes have never been reported before, although the UK Medicines and Healthcare Products Regulatory Agency released an Urgent Field Safety Notice letter in October 2011 with a statement of the manufacturer of the EUS scope (EG3870UTK; Pentax) that balloon channels may not be adequately reprocessed if the balloon remained idle. They issued a warning that it was possible for cross contamination to occur when clogs were formed in the balloon channel. The multiple channels in the scopes are controlled by valves, and it is probable that contamination will spread to other channels through these valves. Therefore, balloons covering the balloon channels are not fully effective in preventing the spread of microorganisms.

Exogenic contaminations by flexible endoscopes were previously described in a review of exogenous endoscopyrelated infections [4]. Not only inadequately decontaminated endoscopes but also inaccurately cleaned accessories and the use of contaminated irrigation fluids or water may lead to deposition of microorganisms in patients and in samples taken for culture. A possible explanation for this kind of contamination is insufficient compliance with disinfecting guidelines. In centres in Canada, moderate compliance with national guidelines was illustrated in a survey on the reprocessing practices of endoscopic retrograde cholangiopancreatography duodenoscopes [5]. In this report only $43 \%$ of centres showed total compliance. Decontamination processes should be monitored and improved on when necessary, as was illustrated for instance in a study that showed diminished viral nucleic acid contamination after changing the protocol for manual cleaning [6].

\section{Disinfectant}

An important factor in the persistence of S. maltophilia seems to be the disinfectant, as was shown by the soiling tests that yielded negative cultures after disinfection with GA but not with PAA.

In the guidelines, both PAA and GA are mentioned as effective disinfectants for the decontamination of endoscopes [7]. Indeed, we achieved effective disinfection of bronchoscopes with both disinfectants; however, disinfection of ultrasound scopes with PAA was inadequate. PAA is believed to have similar or even better biocidal activity and shorter immersion times compared to GA. Another advantage of PAA is that it prevents biofilm for- 
mation. GA enables the creation of biofilm when manual cleaning is inadequate [8]. In the previously cited Canadian survey, samples from centres that had used GA contained higher soil levels with significantly more protein, carbohydrate and endotoxin compared to centres that had used PAA. Cultures of patient-ready flexible duodenoscopes were taken from the biopsy channels and microbial overgrowth (especially S. maltophilia) was present in $7 \%$ of 119 samples [5]. In this survey, the temperatures at which the participating centres had disinfected their scopes were not clear, but at the time valid guidelines recommended disinfecting temperatures of $50-55^{\circ} \mathrm{C}$ for PAA and room temperature for GA [6]. In four different centres (three disinfecting with GA and one with PAA) gastroscope cultures had been taken after decontamination. The centre that had disinfected with PAA at 55$60^{\circ} \mathrm{C}$ showed persistent bacteria in $15 \%$ of cultures.

\section{Temperature}

In many countries, PAA is used at lower temperatures instead of GA at higher temperatures for reasons of staff safety and occupational hygiene. It may well be possible that the temperature of $57-62^{\circ} \mathrm{C}$ used with GA (compared to $25-35^{\circ} \mathrm{C}$ for PAA) was the additional factor that killed S. maltophilia present in niches where, due to the construction of the endoscopes, disinfectants were unable to reach active concentrations. This is supported by the results of the soiling tests with hot water without disinfectant; however, on this subject the literature is inconclusive. Although a superior level of disinfection was shown for 0.125 and $0.27 \%$ GA at temperatures of 55 and $60^{\circ} \mathrm{C}$ compared to $2 \% \mathrm{GA}$ at $20^{\circ} \mathrm{C}$, this effect was not clear with PAA $\left(20^{\circ} \mathrm{C}\right.$ compared to $\left.56^{\circ} \mathrm{C}\right)$ [9]. However, this study was performed on gastroscopes, colonoscopes and duodenoscopes but not on ultrasound scopes.

Storage Time and Humidity of Dryer Air

After prolonged storage of EUS endoscopes in the drying cabinet, cultures were found to be positive after disinfection with PAA, but not after GA disinfection. This observation supports the possibility that a few remaining bacteria may multiply to significant numbers when the endoscope is stored after incomplete disinfection. Obviously, humid compressed air is an important causal factor. This is a clinically relevant observation, since ultrasound scopes are generally not used during weekends.

The reprocessing guidelines provide no uniformity, although endoscope drying is considered to be of great importance to decrease the risk of transmission of waterborne microorganisms [10]. Flushing internal lumens with alcohol could be an effective drying method; however, manufacturers of ultrasound endoscopes do not advocate this method because the materials the scopes are made of are not alcoholproof.

\section{Immersion Time}

In previous studies, the influence of immersion time was discussed. The number of bacteria-free endoscopes increased notably when the exposure time to 2\% GA at $20^{\circ} \mathrm{C}$ was prolonged from 10 to $30 \mathrm{~min}$ [9]. The differences in immersion time in our study (5 and $10 \mathrm{~min}$ for GA and PAA, respectively) cannot explain the fluctuations in the degree of disinfection.

\section{Rinse Water}

The rinse water used for all disinfectors was cultured repeatedly, but $S$. maltophilia was never isolated. Even the disinfecting agents themselves were cultured and they too were found to be negative. So contaminated rinse water (or disinfectant) was not the cause of the infections.

\section{S. maltophilia}

S. maltophilia is a Gram-negative rod that represents colonization rather than infection when isolated from patients. It is the main cause of inflammation in immunocompromised hosts and hospitalized patients, presenting particularly as bloodstream infections or pneumonia [11].

With regard to endoscopes, the ability of S. maltophilia to survive in biofilms is of particular concern. Biofilms accumulate in the channels of endoscopes and these biofilms hold microorganisms together and provide an environment in which many microorganisms proliferate. The effects of pulmonary contamination by endoscopy (i.e., an event where a patient does not develop clinical symptoms) on health is unknown. In our population, S. maltophilia did not contribute to the development of any diseases in our patients. On the other hand, there is the risk of unnecessarily treating patients for presumed infections diagnosed by a sample contaminated by the colonized channels of the endoscope.

\section{Changes in Reprocessing Procedure}

The reprocessing procedure for ultrasound endoscopes was changed by reassigning all endoscopes to fixed locations in the drying and storage cabinet and by using disposable connecting tubes. The compressed air supply was changed from regular to medical grade compressed air with decreased humidity. Furthermore, we switched from PAA to GA as disinfecting agent. It should also be 
mentioned that in our disinfectors GA is used at a higher temperature than PAA, as recommended by the manufacturer. These measures proved to be effective, because from that moment onwards the cultures routinely taken after reprocessing never revealed any $S$. maltophilia.

However, the most logical and probably the most effective solution would be the production of ultrasound scopes without balloon channels. The balloon channels were designed to fix the scope at the moment of tissue aspiration in certain endoluminal positions, but in daily practice they are hardly ever used for this purpose.

The contamination risk of the elevator channel remains a concern, in spite of all the measures that were taken. A redesign of the ultrasound endoscopes could probably solve the contamination problems, and it is the responsibility of the manufacturers to accomplish this new design to improve safety.

\section{Limitations}

It is difficult to determine the extent to which incubation with artificial S. maltophilia-infected soil resembles the real-life situation. Although the same microorganism was used that had been isolated during the pseudo-outbreak, there are always differences compared to the reallife situation.

The concentrated artificial soil probably contains a much higher load of microorganisms than body fluids do, and it is unlikely that body fluids contain a monoculture of bacteria. The test soil stayed in the channels for just 1 day before disinfection took place. In real life, clots of bacteria may remain much longer in the scope channels when they have not been cleaned adequately and may then concentrate more than test soil. Finally, when a scope is contaminated when used in a patient, it is unlikely that all channels will become soiled.

The soiling test was in fact a one-sided test. If the cultures had stayed negative, no conclusions could have been drawn. In this case the cultures remained positive after soiling and disinfecting; this strongly supports the premise that ultrasound endoscopes can act as a reservoir for microorganisms when they have not been adequately disinfected with the agreed disinfector and PAA.

Although the results are suggestive, the significance of the degree of humidity of the drying air remains uncertain since we were not able to repeat the deliberate soiling tests in different air conditions.

\section{Conclusions}

Disinfection of ultrasound endoscopes with PAA (at $25-35^{\circ} \mathrm{C}$ ) is inadequate. Residual S. maltophilia may grow out subsequently during prolonged storage in a relatively humid environment. By using GA (at $57-62^{\circ} \mathrm{C}$ ) instead of PAA as disinfecting agent, we obtained adequate disinfection.

Bronchoscopes were cross-infected via connecting tubes that were contaminated by ultrasound endoscopes. The cross-infection problems were solved by assigning endoscopes to fixed locations in the drying and storage cabinet and by using disposable connecting tubes.

\section{Financial Disclosure and Conflicts of Interest}

The authors declare no conflict of interest.

\section{References}

$\checkmark 1$ Cleaning and disinfection of equipment for gastrointestinal endoscopy. Report of a Working Party of the British Society of Gastroenterology Endoscopy Committee. Gut 1998;42:585-593.

-2 Woodcock A, Campbell I, Collins JV, Hanson PJ, Harvey J, Corris P, Johnston ID: Bronchoscopy and infection control. Lancet 1989; 2:270-271.

$\checkmark 3$ Muscarella LF: Application of environmental sampling to flexible endoscope reprocessing: the importance of monitoring the rinse water. Infect Control Hosp Epidemiol 2002;23:285289.

-4 Seoane-Vazquez E, Rodriguez-Monguio R, Visaria J, Carlson A: Exogenous endoscopyrelated infections, pseudo-infections, and toxic reactions: clinical and economic burden. Curr Med Res Opin 2006;22:2007-2021.

5 Alfa MJ, Olson N, DeGagne P, Jackson M: A survey of reprocessing methods, residual viable bioburden, and soil levels in patientready endoscopic retrograde choliangiopancreatography duodenoscopes used in Canadian centers. Infect Control Hosp Epidemiol 2002;23:198-206.

6 Guideline for the use of high-level disinfectants and sterilants for reprocessing of flexible gastrointestinal endoscopes. Society of Gastroenterology Nurses and Associates, Inc. Gastroenterol Nurs 1999;22:127-134.

7 Ayliffe G: Decontamination of minimally invasive surgical endoscopes and accessories. J Hosp Infect 2000;45:263-277.
$>8$ Rey JF, Kruse A, Neumann C: ESGE/ ESGENA technical note on cleaning and disinfection. Endoscopy 2003;35:869-877.

$\checkmark 9$ Bordas JM, Marcos-Maeso MA, Perez MJ, Llach J, Gines A, Pique JM: GI flexible endoscope disinfection: 'in use' test comparative study. Hepatogastroenterology 2005;52:800807

10 Muscarella LF: Inconsistencies in endoscopereprocessing and infection-control guidelines: the importance of endoscope drying. Am J Gastroenterol 2006;101:2147-2154.

-11 Abbott IJ, Slavin MA, Turnidge JD, Thursky KA, Worth LJ: Stenotrophomonas maltophilia: emerging disease patterns and challenges for treatment. Expert Rev Anti Infect Ther 2011;9:471-488. 